\title{
Control of neuronal branching by the death receptor CD95 (Fas/Apo-1)
}

\author{
C Zuliani ${ }^{1}$, S Kleber ${ }^{1}$, S Klussmann ${ }^{1}$, T Wenger ${ }^{2}$, \\ M Kenzelmann ${ }^{3}$, N Schreglmann ${ }^{1}$, A Martinez ${ }^{4}$, JA del Rio ${ }^{4}$, \\ E Soriano ${ }^{4}$, P Vodrazka ${ }^{5}$, R Kuner ${ }^{5}$, H-J Groene ${ }^{3}$, I Herr ${ }^{2}$, \\ PH Krammer ${ }^{1}$ and A Martin-Villalba ${ }^{\star, 1}$ \\ ${ }^{1}$ Division of Immunogenetics, German Cancer Research Center, INF 280, \\ 69120 Heidelberg, Germany \\ ${ }^{2}$ Clinical Cooperation Unit Molecular Oncology/Pediatrics, German Cancer \\ Research Center, INF 280, 69120 Heidelberg, Germany \\ ${ }^{3}$ Cellular and Molecular Pathology, German Cancer Research Center, 69120 \\ Heidelberg, Germany \\ 4 Developmental Neurobiology and Regeneration Group, Scientific Park of \\ Barcelona, Josep Samitier, Barcelona, Spain \\ 5 Department of Pharmacology, University of Heidelberg, Heidelberg Germany \\ * Corresponding author: A Martin-Villalba, Tumor Immunology, Division of \\ Immunogenetics, DKFZ Heidelberg, INF 280 Heidelberg 69120, Germany. \\ Tel: + 496221 423766; Fax: + 496221411715 ; \\ E-mail: a.martin-villalba@dkfz.de
}

Received 28.4.05; revised 06.6.05; accepted 07.6.05; published online 08.7.05 Edited by P Nicotera

\begin{abstract}
The CD95 (Apo-1/Fas)/CD95 ligand (CD95L) system is best characterized as a trigger of apoptosis. Nevertheless, despite broad expression of CD95L and CD95 in the developing brain, absence of functional CD95 (Ipr mice) or CD95L (gld mice) does not alter neuronal numbers. Here, we report that in embryonic hippocampal and cortical neurons in vivo and in vitro CD95L does not induce apoptosis. Triggering of CD95 in cultured immature neurons substantially increases neurite branches by promoting their formation. The branching increase occurs in a caspase-independent and death domain-dependent manner and is paralleled by an increase in the nonphosphorylated form of Tau. Most importantly, Ipr and gld mutants exhibit a reduced number of dendritic branches in vivo at the time when synapse formation takes place. These data reveal a novel function for the CD95 system and add to the picture of guidance molecules in the developing brain.
\end{abstract}

Cell Death and Differentiation (2006) 13, 31-40.

doi:10.1038/sj.cdd.4401720; published online 8 July 2005

Keywords: CD95 (Fas/Apo-1); apoptosis; neurite remodeling; branching; brain development

Abbreviations: $\mid p r$, lymphoproliferative; gld, generalized lymphoproliferation; TUNEL, terminal dUTP nick end labeling; LZ-CD95, leucine-zipper-CD95L; DIV, days in vitro; MTs, microtubules; $\mathrm{Hc}$, hippocampus; Cx, cortex

This paper is dedicated to M. Hergenhahn (

\section{Introduction}

The CD95 (APO-1/Fas) ligand (CD95L) induces apoptosis upon binding to CD95. This holds true for the injured adult brain (e.g. in stroke and spinal cord injury), where neural and glial cells are cleared via CD95-mediated apoptosis. ${ }^{1-3}$ Ligation of CD95 by trimerized CD95L leads to recruitment of the adapter protein Fas-associated death domain (FADD), MORT1 $)^{4}$ and caspase- 8 into a death-inducing signaling complex (DISC). ${ }^{5}$ FADD contains a death domain (DD) and a death-effector domain (DED). Via its DD, FADD binds to the DD of CD95. ${ }^{6}$ The DED recruits the DED-containing procaspase-8 into the DISC. ${ }^{5}$ Procaspase- 8 at the DISC is activated through self-cleavage and commits the cell to apoptosis by activation of downstream effector caspases. ${ }^{7}$ An additional pathway downstream of CD95 has been described in dorsal root ganglia cells (DRGs). In these cells triggering of the CD95 leads to DD-independent activation of ERK that finally results in axonal elongation and not in apoptosis. ${ }^{8}$

Expression of CD95L and CD95 is also found in the brain at embryonic and early postnatal stages. ${ }^{9,10}$ Although some studies report on CD95-induced death of embryonic cortical precursor cells, ${ }^{11,12}$ others find these cells to be resistant to CD95-induced death. ${ }^{13,14}$ The fact is that contrary to caspasedeficient mice showing an excess of neurons, ${ }^{15}$ the neuronal density in the hippocampus (Hc) of lymphoproliferative (Ipr) and generalized lymphoproliferation ( $g / d$ ) mice, with functionally deficient CD95 and CD95L, respectively, ${ }^{16}$ was similar to the density in wild-type (wt) animals. ${ }^{17}$ Thus, apoptosis in the developing hippocampus does not seem to be controlled by the CD95/CD95L system.

To examine the role of the CD95/CD95L-system in the developing brain, we first confirmed that these proteins are expressed throughout development and that they do not induce apoptosis in vivo. Dil tracing of CA1 pyramidal neurons revealed less dendritic branches in Iprand gld mice compared to $w t$ animals. Also, triggering of CD95 in cultured neurons increased neuronal branching by promoting the formation of new neurites. In addition, our results suggest that CD95 mediates neuronal branching via its DD. Thus, CD95 regulates neurite remodeling.

\section{Results}

\section{CD95 and CD95L are expressed in the developing brain}

To quantify the levels of CD95 and CD95L protein expression at different stages of development in the brain, we first performed fluorescence-activated cell sorter (FACS) analysis. To control specificity of the different antibodies, we used a murine thymoma cell line known to express CD95 (E20) and a murine melanoma cell line stably transfected with cd95I (B16cd95) (Figure 1a). In the developing hippocampus and cortex 


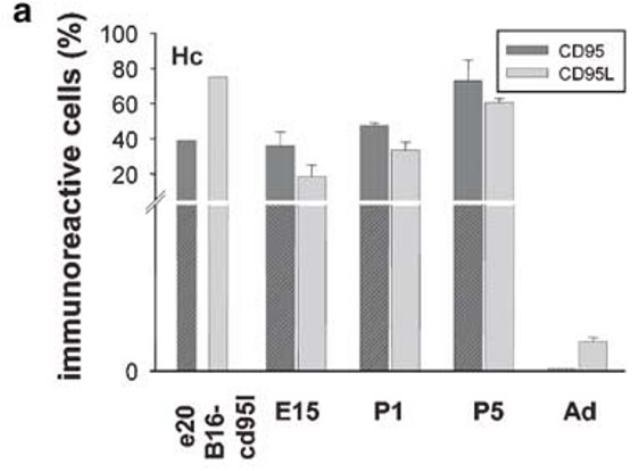

b
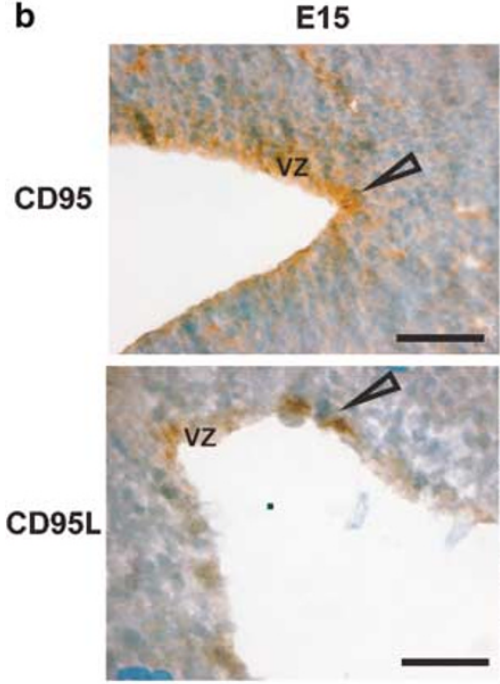

C

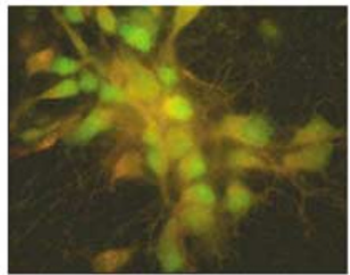

NeuN/CD95L

P1

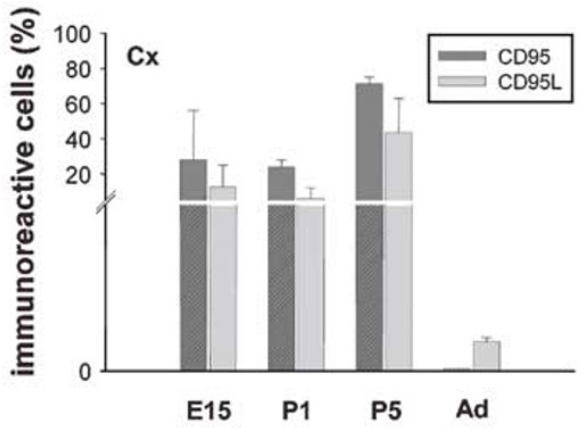

P1

Control
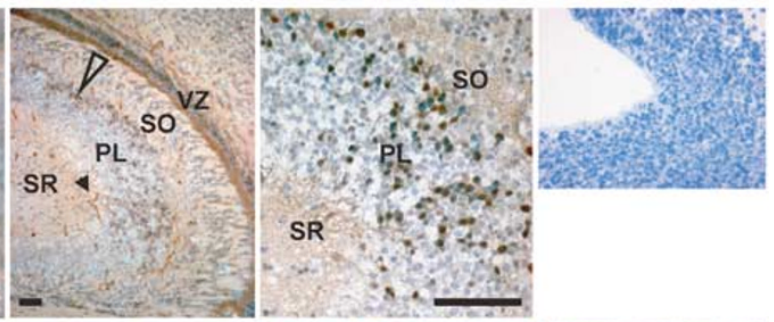

$\mathrm{SR}$
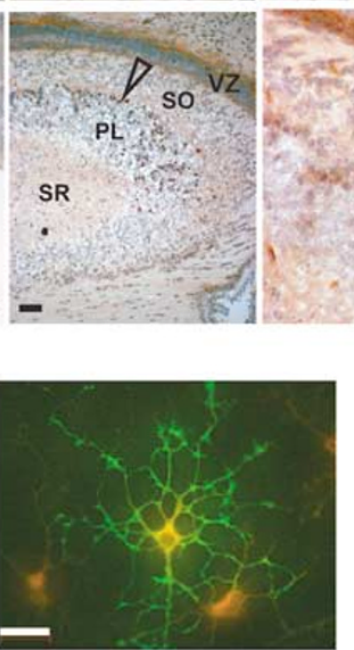

CNPase/CD95L

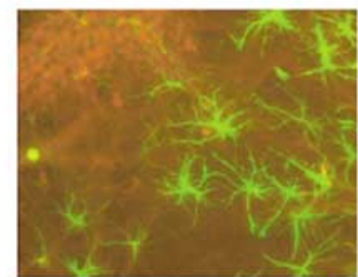

GFAP/CD95L

Figure 1 CD95L and its receptor are expressed throughout development. (a) Surface expression of CD95 and CD95L in the hippocampus and cortex was quantified by FACS analysis. CD95-expressing murine thymoma cells (E20) and cd95/-transfected murine melanoma cells were used as controls. (b) Immunoreactivities of CD95, CD95L and secondary antibody alone (control) in E15 and P1 mouse brains. Filled arrow show endothelial staining; bold arrows show immunoreactivities of the progenitor cells in the VZ in E15 mice and of the neuronal layers of the hippocampus in P1 mice. (c) Photomicrographs of hippocampal explants (1 week in vitro) showing double staining with an antibody detecting CD95L (N20) and antibodies specific for neurons (NeuN), oligodendrocytes (CNPase) or astrocytes (GFAP). Results are expressed as mean \pm S.E. and acquired as triplicates of three independent experiments. Hc: hippocampus; Cx: cortex; E15: embryonic day 15; P1,P5: postnatal day 1,5; SLM: stratum lacunosum-moleculare; SR: stratum radiatum; SO: stratum oriens; PL: pyramidal layer; VZ: ventricular zone. Scale bar: $50 \mu \mathrm{m}$

(Cx), CD95 and CD95L were already expressed at embryonic day 15 (E15), reaching maximal levels at postnatal day 5 (P5; Figure 1a). In the adult brain, CD95 and CD95L were barely detectable (Figure 1a).

To further substantiate the pattern of CD95L and CD95 expression, we performed immunohistochemistry. Consistent with the above results, CD95L immunoreactivity was detected between $\mathrm{E} 16$ and $\mathrm{P} 5$ in the subventricular zone, in the cortical plate of the neocortex and in the neuronal layers of the hippocampus (Figure 1b). Immunostaining at E15 was most prominent in the subventricular zone. At P1-P5 stages, the staining was strongest in the neuronal layers of the hippocampus and in the cortical layers II-III and V of the neocortex, where pyramidal neurons were intensely labeled (Figure 1b). The overall pattern of CD95 immunoreactivity during the brain development was similar to that of CD95L (Figure 1b). The intensity of staining also increased at P5 in both hippocampal and neocortical neurons. In these regions the immunoreactivity was most prominent in pyramidal neurons of the hippocampal pyramidal layer and in layers 
II-III and VIb (Figure 1b). From P1 onwards, immunoreactivity for CD95 and CD95L was also found in endothelial cells. In the adult brain CD95 and CD95L were barely present, and if present, immunoreactivity was restricted to endothelial cells (data not shown).

To identify the cells in charge of producing CD95L, we performed double staining on hippocampal explants cultured for 1 week. CD95L was detected in neurons and oligodendrocytes, but not in astrocytes (Figure 1c).

\section{Triggering of CD95 in immature neurons does not induce apoptosis}

We next addressed whether stimulation of CD95 leads to apoptosis in the developing central nervous system (CNS). Lentiviral vectors containing the green fluorescent protein (GFP) and cd95/ were injected intracortically in P4 brains (Figure 2a). At P9, the rate of apoptosis was assessed by terminal dUTP nick end labeling (TUNEL). In parallel, the ability of the lentivirally expressed CD95L to induce apoptosis was confirmed in P693 cells (data not shown). At these stages, overexpression of cd95I did not induce apoptosis in vivo (Figure $2 \mathrm{~b}$ ).

Also, cultured cortical neurons were treated with LeucineZipper (LZ)-CD95L (100 ng/ml). Apoptosis was assessed $24 \mathrm{~h}$ afterwards by Nicoletti staining. Only after 6 or more days in vitro (DIV), neurons became sensitive to CD95L-induced apoptosis ( $\sim 20 \%$ apoptosis; Figure $2 \mathrm{c}$ ). At earlier time points, treatment with CD95L did not affect neuronal viability (Figure 2c).

\section{CD95L induces branching in early stage hippocampal neurons}

Hippocampal neurons are highly polarized and are broadly used as a model system to study development-induced morphological changes. In these neurons, we further checked whether CD95L induced any morphological changes. Upon treatment with CD95L $(100 \mathrm{ng} / \mathrm{ml})$ no changes in the average neurite length or in the number of neurites were observed (see Supplementary Figure 1a). Similarly, in hippocampal explants CD95L did not influence the average length of neurites (data not shown). Importantly, in hippocampal neurons, CD95L significantly increased the number of branching points per cell (Figure 3a, b). The increase in branching points by CD95L was significant in the axonal and in the dendritic compartment (Figure 3b). To exclude a nonspecific influence of the LZCD95L preparation, we neutralized the activity of LZ-CD95L with an anti-CD95L antibody (NOK-1). LZ-CD95L-induced branching was blocked by NOK-1 (see Supplementary Figure 1b). Likewise, triggering of CD95 by an agonistic antibody increased the number of global (axonal and dendritic) branching points (data not shown).

Neurons deficient in functional CD95 (Ipr) or CD95L ( $g / d$ ) developed normally and the number of branching points per cell at 4 DIV is comparable to wt neurons (Figure $3 b$, $d$ and data not shown). Likewise, acute neutralization of CD95L activity in wt neurons did not alter neuronal global branching in a

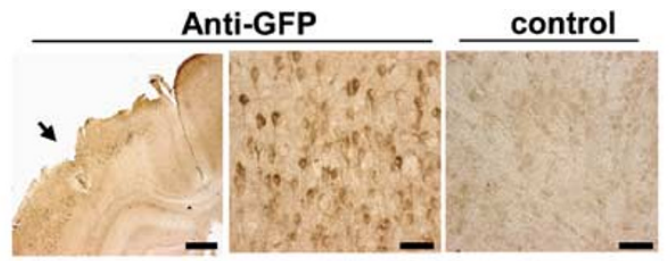

b
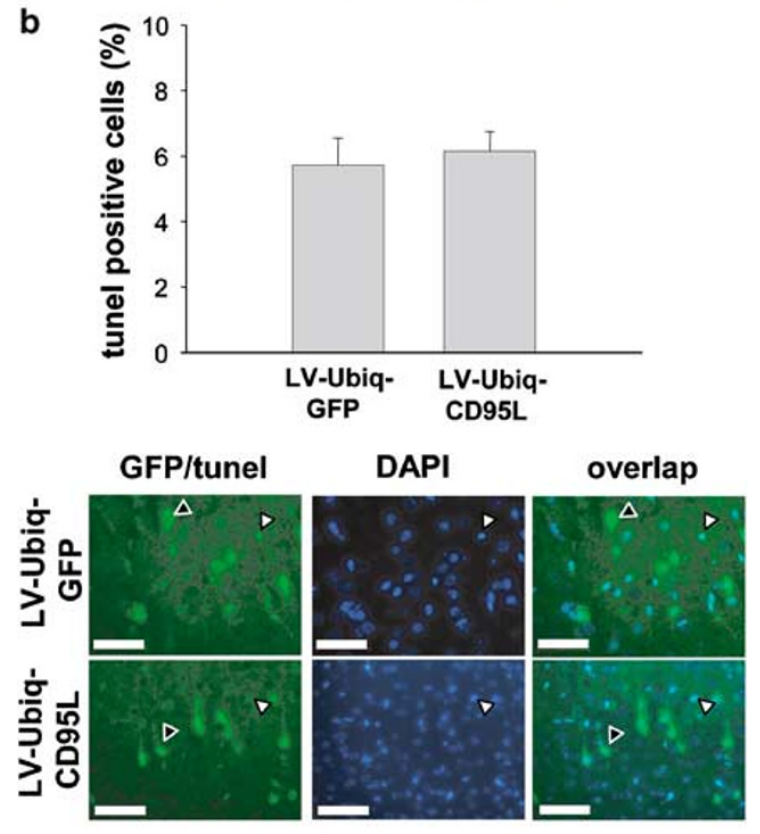

C

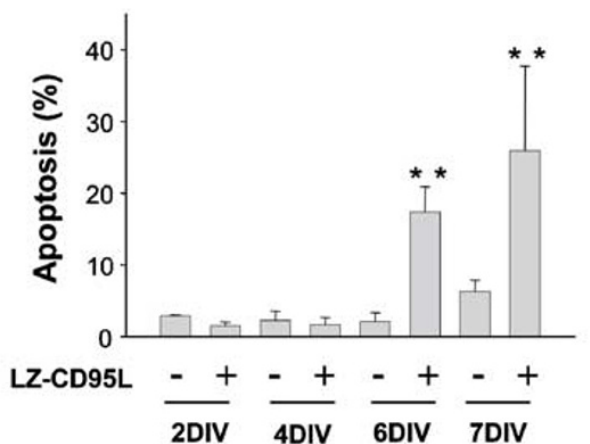

Figure 2 Triggering of CD95 in immature neurons does not induce apoptosis. Lentiviral constructs of GFP and CD95L (LV-Ubiq-GFP and LV-Ubiq-CD95L, respectively) were injected in the cerebral cortex of $\mathrm{P} 4$ mice. (a) Representative pictures of GFP immunoreactivity showing the Lentiviral distribution in the cortex of P9 mice. Sections were incubated without the primary antibody as a control. The arrow indicates the injection site. (b) In brain sections from P9 mice apoptosis was detected by TUNEL and expressed as the percentage of total cells (as assessed by DAPI counterstaining). Representative photomicrographs of P9 brains showing GFP/TUNEL staining (left), DAPI (center) and merge (right panel). The black arrows indicate GFP-positive neurons. The white arrows show cells labeled for TUNEL and exhibiting a condensed nucleus with DAPI. (c) Neurons at 2, 4, 6 and 7 days in vitro (DIV) were treated with LZ-CD95L or left untreated for $24 \mathrm{~h}$. Thereafter, DNA fragmentation was measured by FACS analysis. Results are expressed as mean \pm S.E. ${ }^{* *} P<0.001$ and acquired as triplicates in three independent experiments. Scale bar: $500 \mu \mathrm{m}(\mathrm{a}$, left), $50 \mu \mathrm{m}$ ( $a$, center and right; b)

4 DIV neurons as compared to neurons treated with control IgG or left untreated (see Supplementary Figure 1c).

Co-cultures with either hippocampal explants or highdensity cortical cultures increased global branching of 
a

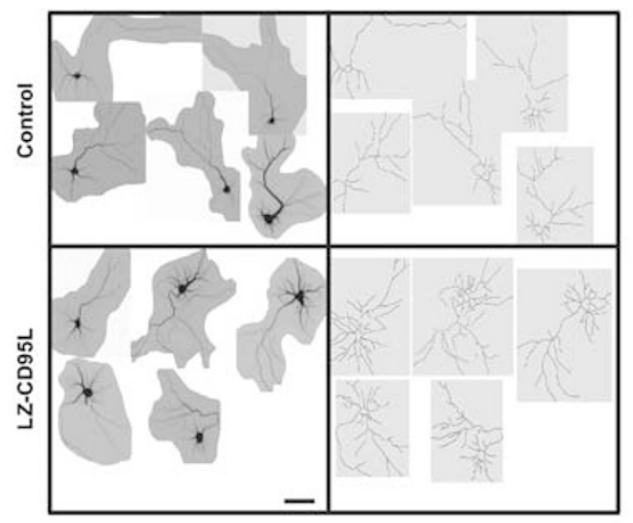

b

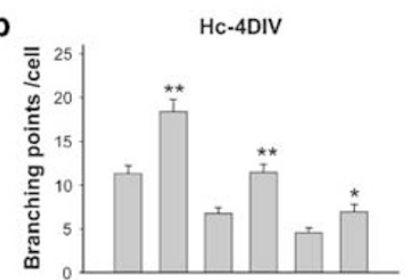

LZ-CD95L $\frac{-+}{\text { total }} \frac{-+}{\text { axon }}+\frac{+}{\text { dendrites }}$
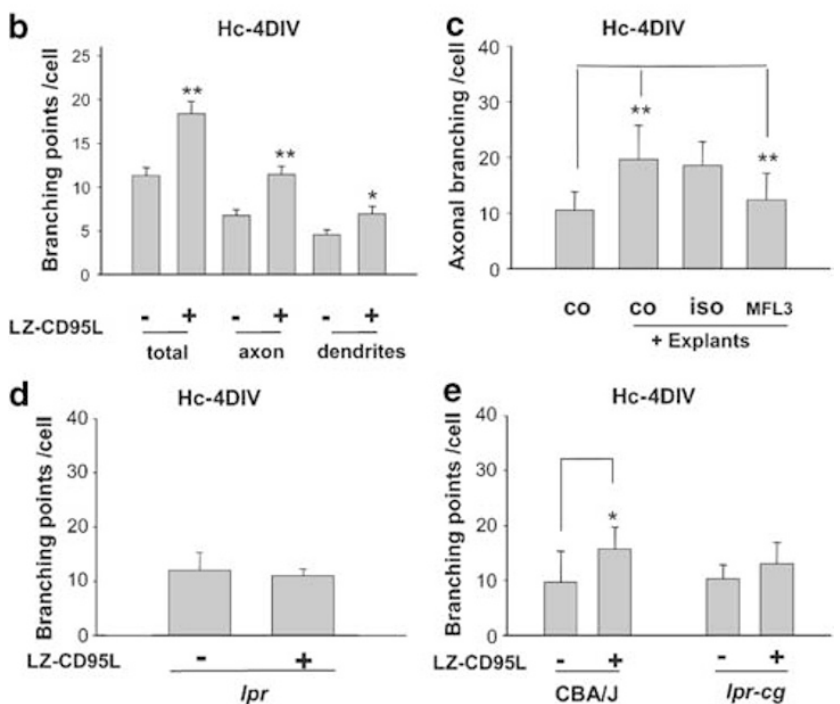

Figure 3 CD95L induces branching in early-stage hippocampal neurons. (a) CD95L significantly increases the number of branching points per cell in $\mathrm{E} 15 \mathrm{wt}$ hippocampal neurons $(\mathrm{Hc})$ at 4 DIV. Fluorescence images and representative drawings are shown. (b) The increase in branching was found in the axons and dendrites of wt mice. (c) Coculturing of $\mathrm{Hc}$ neurons with $\mathrm{Hc}$ explants increased axonal branching in the isolated $\mathrm{Hc}$ neurons. This effect was blocked by a neutralizing antibody to CD95L (MFL3) but not by the isotype control antibody (iso). (d, e) CD95L failed to induce branching in 4 DIV Hc neurons from $\operatorname{lpr}$ (d) and lpr-cg (e) mice. CBA/J neurons were used as wt because lpr-cg mice are on a CBA/J background (e). Data are expressed as mean \pm S.D. ${ }^{* \star} P<0.001$ or ${ }^{*} P<0.05$. Results are representative of three independent experiments. Hc: Hippocampus; DIV: days in vitro. Scale bar: $50 \mu \mathrm{m}$

hippocampal neurons (Figure $3 \mathrm{c}$ and data not shown). Importantly, branching could be partly inhibited by a neutralizing antibody to CD95L (MFL3; Figure 3c). These data show that endogenous $\mathrm{CD} 95 \mathrm{~L}$ induces branching in the embryonic brain. Further, in neurons from Iprand /pr-cg (Ipr-complementing gld) mice, which express little CD95 or death domainmutated CD95, respectively, ${ }^{18,19}$ treatment with CD95L $(100 \mathrm{ng} / \mathrm{ml})$ did not significantly increase the number of branching points per cell (Figure 3d, e).

\section{Branch dynamics in hippocampal neurons after triggering of CD95 in vitro}

To determine whether CD95L functions to promote the formation of new axonal branches or to inhibit the retraction
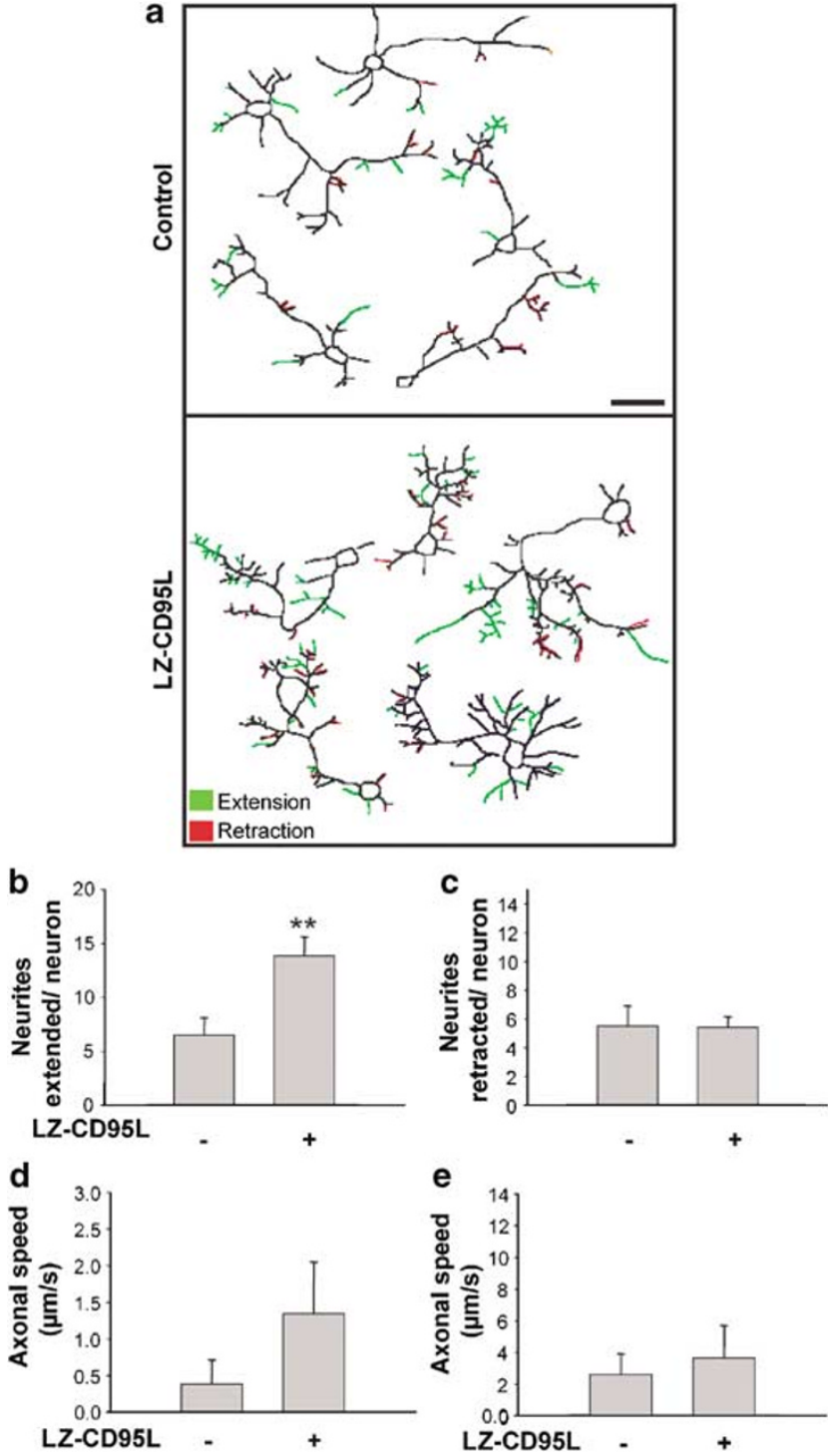

Figure 4 Branch dynamics in hippocampal neurons after triggering of CD95 in vitro. (a-d) Time course recording of the same neuron over $4 \mathrm{~h}$ at $4 \mathrm{~min}$ intervals. (a) Representative drawing of neurons treated with LZ-CD95L or left untreated. Neurites undergoing retraction after $4 \mathrm{~h}$ are shown in red in the $0 \mathrm{~h}$ drawings. The new extended neurites after $4 \mathrm{~h}$ are shown in green in the $0 \mathrm{~h}$ drawings. (b, c) Quantification of the neurites extended (b) or retracted (c) per neuron in the time course experiments. (d, e) Quantification of the axonal advance rate expressed in $\mu \mathrm{m}$ per second at $4 \mathrm{~h}$ (d) and $10 \mathrm{~h}$ (e). Data are expressed as mean \pm S.D. ${ }^{* \star} P<0.001$. Results are representative of three independent experiments. Scale bar: $50 \mu \mathrm{m}$

and pruning of previously formed branches, we monitored branch dynamics of cultured hippocampal neurons between 3 and 4 DIV by time-lapse microscopy. Treatment with LZCD95L $(100 \mathrm{ng} / \mathrm{ml})$ resulted in a prominent increase in the number of new branches, whereas the number of branches undergoing spontaneous retraction was not altered (Figure $4 a-d)$. In addition, the rate of axonal growth was increased after $4 \mathrm{~h}$ (Figure $4 \mathrm{~d}$ ) but not after $10 \mathrm{~h}$ (Figure $4 \mathrm{e}$ ) of LZ-CD95L treatment. This indicates a short transient motility increase of generated axons (Figure 4d, e). Thus, triggering of CD95 
increases branching by inducing the formation of new branches.

\section{Branching is not signaled by caspases and transcription factors}

CD95 transduces the apoptotic signal through activation of caspases. To examine whether caspases are required for the induction of neuronal branching, we used a broadspectrum caspase inhibitor, benzoyl-VAD.fluoromethyl ketone (zVAD.fmk) at a dose that blocks caspases but not other proteases $(25 \mu \mathrm{M})$. zVAD.fmk did not block CD95L-mediated neuronal branching (Figure 5a, b). Consistently, the amount of cleaved caspase-3 did not increase upon CD95L treatment (Figure 5c).

Transcription factors (TFs) regulate the expression of numerous genes involved in neuronal branching; therefore, we would expect changes in the regulation of several genes upon triggering of CD95. Using the Affymetrix oligonucleotide array Mu-U74Av2 ( 12.600 seq.) we compared the gene expression profile of CD95L-treated and nontreated neurons. At 1 DIV, no differentially transcribed genes were found (see Supplementary Figure 1d).

The other molecule involved in morphological modifications is the microtubule-associated protein Tau, a member of a group of proteins promoting assembly and stabilization of microtubules (MTs). ${ }^{20,21} \mathrm{An}$ increase in Tau phosphorylation reduces its affinity for MTs, thereby reducing Tau-mediated polymerization and stabilization of MT structures. ${ }^{22,23} \mathrm{We}$ checked the phosphorylation state of Tau by using an antibody (Tau-1) that detects Tau when not phosphorylated at Ser-199/202. The level of dephosphorylated Tau increased upon treatment with CD95L (Figure 5d). Likewise, treatment with alkaline phosphatase increased Tau- 1 immunoreactivity in untreated but not in CD95L-treated neurons (data not shown). Moreover, this effect remained unaffected by zVAD.fmk (Figure 5d)

\section{Mice deficient in functional CD95L or CD95 exhibit reduced dendritic branching in CA1 neurons}

To investigate whether the lack of CD95L signaling affected (dendritic) branching in vivo, we analyzed the dendritic trees of CA1 pyramidal neurons in wt and mutant postnatal mice. To retrogradely label pyramidal neurons, crystals of the lipophylic tracer 1,1'-dioctadecyl-3,3,3',3'-tetramethylindocarbocyanine perchlorate (Dil) were injected into the fimbria of P5 forebrains. 3-D reconstructions of filled neurons suggested that $g l d$ and $l p r$ mutant neurons had reduced dendritic trees compared to wt CA1 neurons (Figure 6a). Indeed, the number of dendritic branching points was reduced by $24-26 \%$ in the hippocampus of gld and Ipr mice compared to their wt counterparts. This reduction was observed in both the basal and apical dendritic trees (Figure 6b). Similarly, at P10, the dendritic trees of Ipr purkinje cells as assessed by calbindin staining exhibited a more diffuse immunoreactivity due to having less and thinner terminal side branches than their wt counterparts (Figure 6c). a
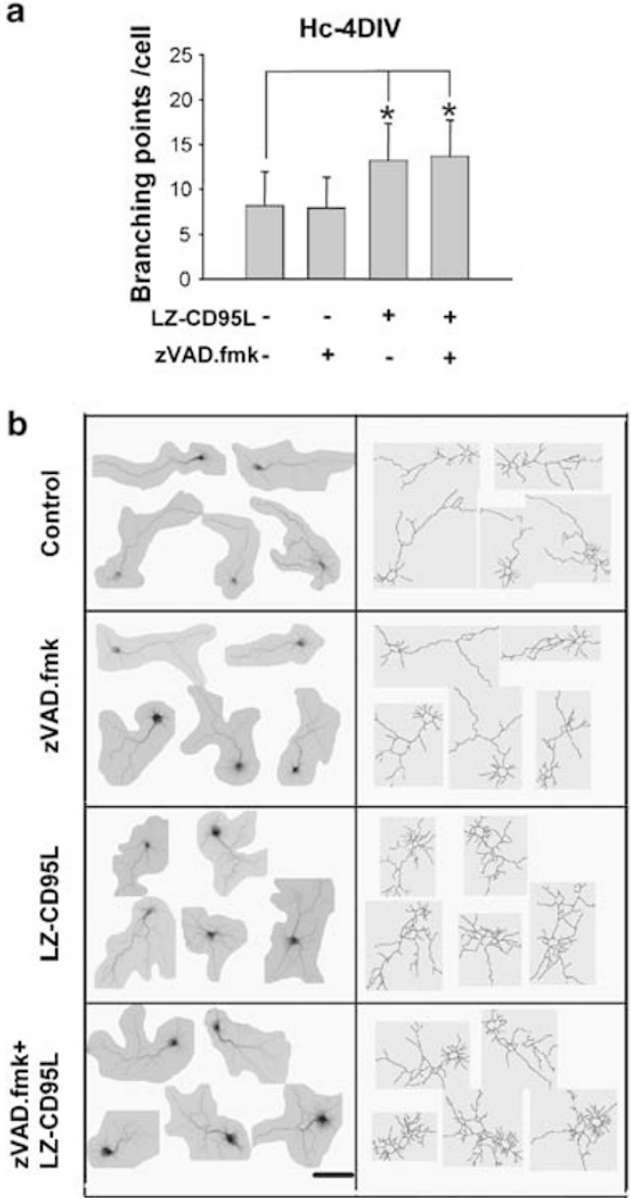

C
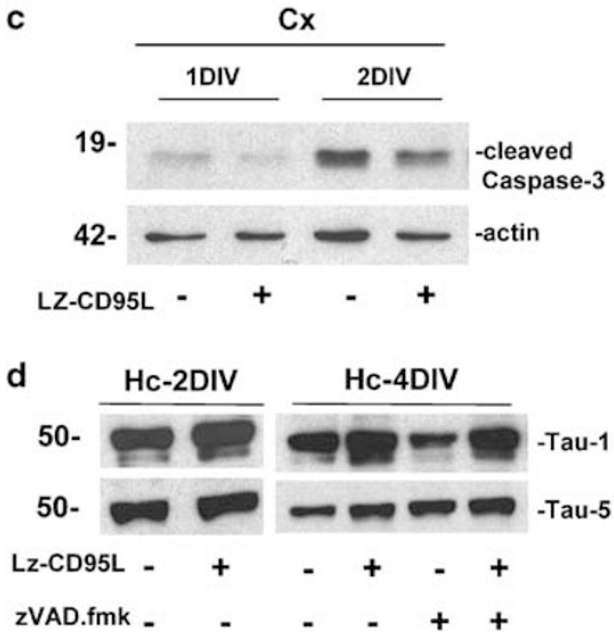

Figure 5 Branching is not mediated by caspases. (a) zVAD.fmk did not block CD95L-induced branching of Hc neurons at 4 DIV. (b) In vitro fluorescence images and representative drawings of neurons quantified in A are shown. (c) Treatment with CD95L did not increase the amount of cleaved caspase-3. (d) The microtubule associated protein Tau showed a higher dephosphorylation state upon CD95L treatment (Ser 199/202 dephosphorylated Tau; Tau-1). This effect was not affected by zVAD.fmk. The total amount of Tau was confirmed using an anti-Tau antibody (Tau-5) recognizing Tau independently of its phosphorylation state.Hc: Hippocampus; Cx: Cortex; DIV: days in vitro. Data are expressed as mean \pm S.D. ${ }^{\star} P<0.05$. Results are representative of three independent experiments. Scale bar: $50 \mu \mathrm{m}$ 
a

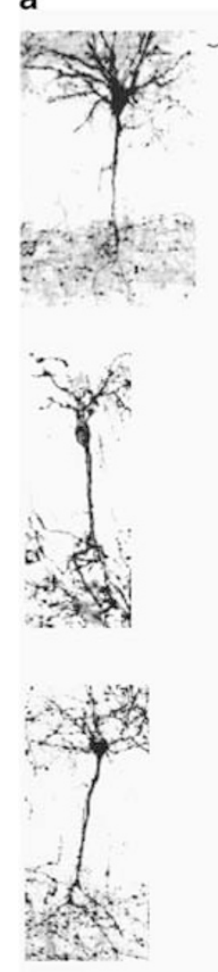

C

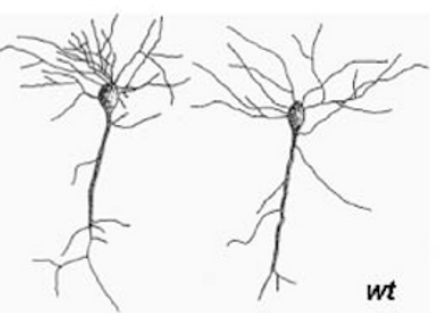

wt
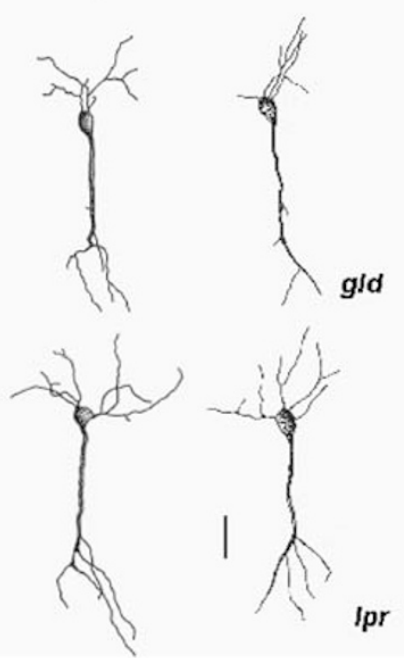

b

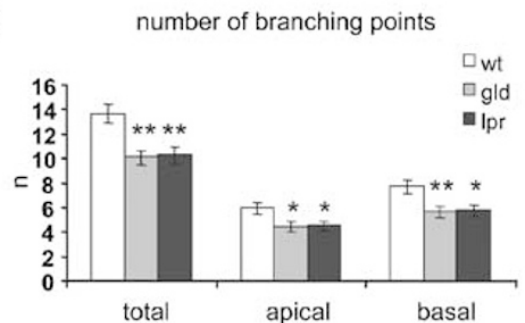

wt
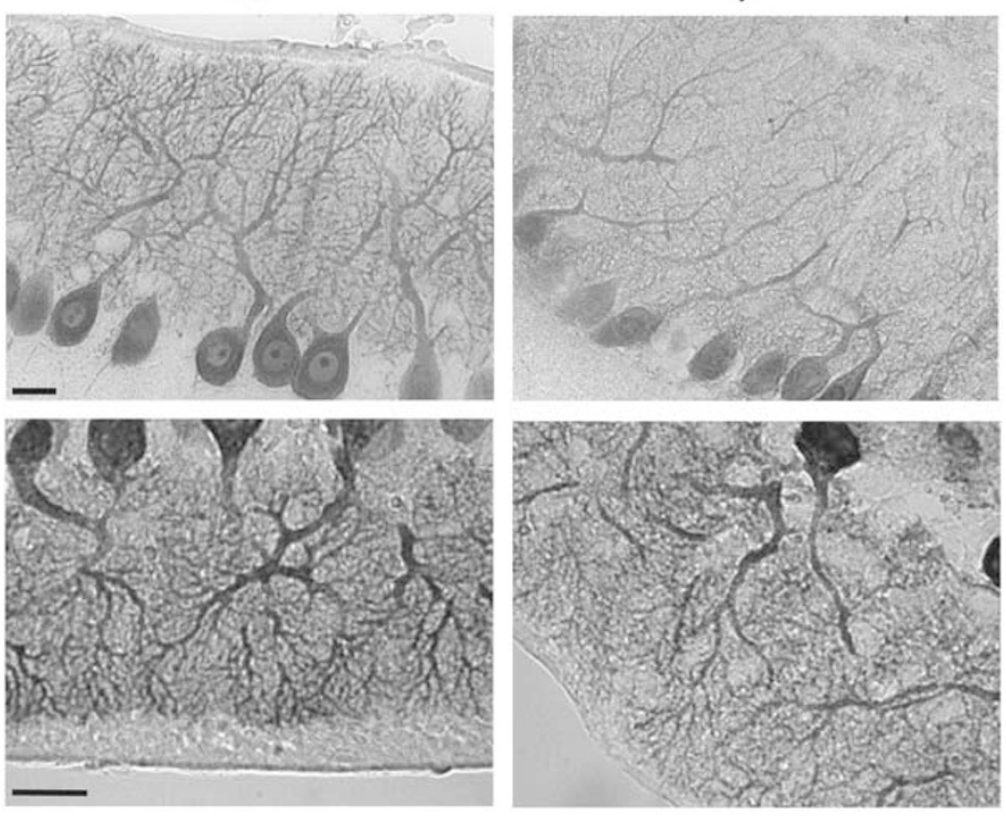

Ipr
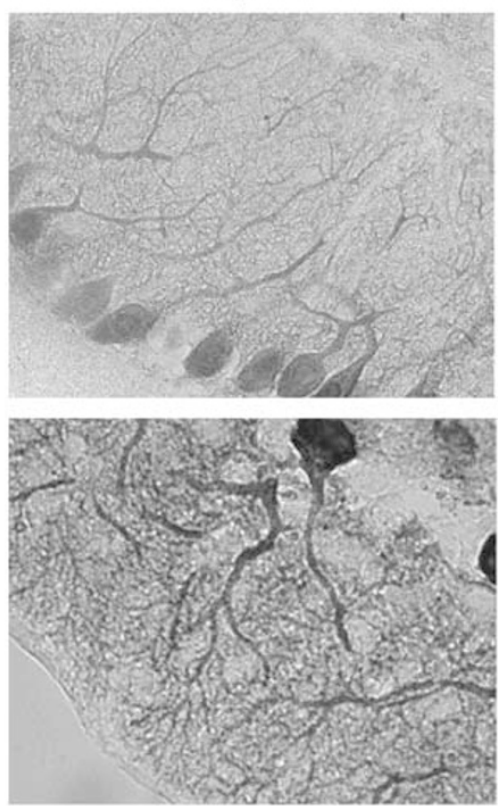

Figure 6 Reduced dendritic trees in the hippocampi of postnatal Ipr and gld mutant mice. (a) Computer-generated (left column) and line-drawing (central and right columns) reconstructions of Dil-labeled CA1 pyramidal neurons of wt, gld and /pr mutant mice. (b) Graphic illustrating the number of branching points in P5 wt, gld and /pr CA1 pyramidal neurons. (c) Representative pictures of the calbindin staining showing a low magnification (upper panel) and a high magnification (lower panel) of the dendritic trees of the Purkinje cells of P10 wt and Ipr mice. Data are shown for basal and apical dendrites, as well as for the entire dendritic tree (mean \pm S.E.M.; * $P<0.05 ;{ }^{* *} P<0.01$, compared to wt neurons). Scale bar: $100 \mu \mathrm{m}(\mathbf{a}) ; 20 \mu \mathrm{m}(\mathbf{c})$

\section{Discussion}

\section{CD95 controls branching of central neurons in vivo and in vitro}

Here we show that the CD95 system induces neuronal branching. Both exogenously added and endogenously produced CD95L increased the number of branching points in cultured hippocampal and cortical neurons. Consistently, in the developing brain, expression of CD95 and CD95L was found in neurons at the appropriate time and location to act in modeling of the dendritic and axonal arbors. The Ipr mutation is caused by insertion of a retrotransposon into the second intron of the cd95 gene, which results in premature termination of transcription and barely detectable levels of the CD95 protein ( $\sim 10 \%$ of normal levels). The absence of normal CD95-induced apoptosis results in prolonged survival of activated lymphocytes and autoreactive T- and B-cell clones, which in turn induce a lupus-like autoimmune disease. ${ }^{24}$ Examination of the hippocampal pyramidal neurons of 5week-old Ipr mice revealed shrinkage of dendritic arborisation and loss of dendritic spines. ${ }^{25}$ Although the lack of a dendritic growth factor(s) was proposed, most of the neurological features were regarded as a consequence of autoimmunity. Here we show that already at P5, when autoimmunity is absent, CA1 neurons of Ipr and gld mutants exhibit reduced numbers of dendritic branching points. Likewise, the dendritic arbors of P10 cerebellar purkinje cells had less terminal branches in Ipr than in wt mice. In vitro, CD95L-mediated branching was more prominent in the axonal than in the dendritic compartment. This observation might partially be explained by the fact that axonal remodeling takes place prior to dendritic remodeling. Although axonal branching was not directly examined in vivo, the observed expression of CD95 
and CD95L in the developing brain and the CD95L-induced increase in axonal branching in vitro suggest a decrease in axonal branching and possibly in synaptic density in the gld and Ipr mice. Nevertheless, further examination is required to confirm this assumption.

However, in neuronal cultures from Ipr, gld or wt mice where CD95L activity was neutralized there was no change in the number of branching points. In these low-density neuronal cultures CD95L is not produced in sufficient amounts to stimulate branching. Increasing the amount of CD95L by using hippocampal explants or high-density cortical cultures increased branching of isolated hippocampal neurons.

\section{CD95-induced branching is neuronal-type specific}

In dorsal root ganglia (DRG) cells, CD95L has been reported to increase neurite outgrowth. ${ }^{8}$ By contrast, in embryonic hippocampal and cortical neurons CD95L did not change the average neurite length. Similarly, the length of the neurites arising from the hippocampal explants was not altered either by exogenously added CD95L or neutralizing antibody to CD95L (data not shown). Also, in primary sensory neurons, CD95L induces neurite outgrowth through activation of ERK. ${ }^{8}$ ERK activation increases expression of $\mathrm{p} 35$, a neuron-specific activator of cyclin-dependent kinase 5 (cdk5). Inhibition of ERK prevented p35 upregulation and thereby neurite outgrowth. ${ }^{8}$ In hippocampal and cortical neurons, however, inhibition of ERK phosphorylation by the MEK inhibitor PD98059 did not alter CD95L-induced branching (see Supplementary Figure 2a). Also, treatment with CD95L did not activate ERK or alter expression of p35 (Supplementary Figure $2 \mathrm{a}$ and data not shown). In summary, these data indicate that ERK does not mediate the increase in branching reported here. Altogether, these data indicate that the function and signaling downstream of CD95 in sensory neurons is distinct from that in central neurons and, therefore, neuronaltype specific. Consistently, CD95L failed to induce branching in motor neurons but instead induced apoptosis (Raoul et al ${ }^{26}$ and data not shown).

\section{CD95-induced branching is DD dependent and caspase independent}

This DD-dependent and caspase-independent CD95-induced effect is also found in T cells. ${ }^{27}$ Activated CD95 recruits FADD via its $D D$, which in turn recruits other molecules through its DED domain. Currently, the only proteins known to bind to the DED domain of FADD are procaspase-8, -10 and FLIP. Whether FLIP or another unknown DD/DED-bearing molecule couples CD95 to a branching pathway remains to be studied. Differently, CD95L-induced neurite outgrowth of DRG cells is DD independent and not sensitive to caspase inhibitors. ${ }^{8}$ This might help explain why in these two different neuronal populations activated CD95 triggers different signaling pathways.

CD95 might regulate neuronal branching by changing the phosphorylation state of cytoskeletal targets such as the microtubule-associated protein Tau. ${ }^{28}$ Tau binds to microtubules in a phosphorylation-dependent manner, and this interaction contributes to the stability of microtubules and, thus, to changes in neuronal morphology. Consistently, we found higher levels of dephosphorylated Tau (Ser 199/202) upon treatment with CD95L. Other targets of CD95 might be transcription factors that modulate several other genes. However, at 1 DIV, no differentially expressed genes at the transcriptional level could be detected. In view of mRNA storage in neurons, ${ }^{29}$ this might indicate either that all changes were due to differences in protein-protein interactions or to changes in the translation state of already existing mRNAs.

\section{CD95, apoptosis and neuronal plasticity}

Brain expression of CD95 and CD95L is apparent during development and disease, but is otherwise barely detectable. Our studies have shown that CD95 promotes neurite remodeling during development and apoptosis during disease. ${ }^{1-3}$ It seems nevertheless possible that the presence of CD95 and CD95L in the injured mature nervous system may also be required to enable synaptic reorganization processes that accompany spontaneous neuronal plasticity. We hypothesize that factors present in the injured CNS such as reactive oxygen species, increased lactate or decreased ATP levels might sensitize the cell to CD95-induced apoptosis. Alternatively, in the developing brain the presence of other extracellular factors, such as neurotrophins, slits, ephrins and integrins might prone cells to undergo synaptic reorganization in the presence of CD95L. Knowledge of the factors determining whether the cell undergoes apoptosis or neuronal plasticity might uncover new therapeutic strategies for neurodegenerative diseases.

\section{Lack of CD95 leads to neurobehavioral deficits}

Defects in CD95 and CD95L in mice result in marked overaccumulation of mature lymphocytes and autoimmune disease. ${ }^{19,30}$ Moreover, defective lymphocyte apoptosis caused by mutations of the cd95 gene can result in a severe autoimmune lymphoproliferative syndrome (ALPS) in humans. ${ }^{31}$ In the mouse model for ALPS cognitive and neurological deficits were detected in 16-week-old mice. ${ }^{32}$ These neurological deficits were assessed by the water maze test and other standardized neurological rodent examinations. A significant increase in anti-phospholipid antibodies (aPL) was proposed to be the reason for the cognitive and neurological deficits. However, no correlation of these symptoms with aPL titers could be found in these mice. ${ }^{32}$

In human patients with ALPS, autoimmune manifestations like systemic lupus erythematosus (SLE) are the second most frequent event. In SLE, central nervous system complications occur in up to $75 \%$ of patients. These complications include seizures, stroke, psychosis, transverse myelitis and chorea. $^{33,34}$ There is also evidence that a subgroup of SLE patients have cognitive impairments, including some without clinically obvious neuropsychiatry symptoms. ${ }^{35,36}$ Also in humans, an increase in aPL was proposed to be the reason for the cognitive impairment. Nevertheless, there is no convincing data that confirms this hypothesis. ${ }^{35,37,38}$ Published data on mice and humans and our own data obtained 
from mice suggest that neurological deficits found in Ipr mice are not a consequence of autoimmunity but a primary consequence of a nonfunctional CD95/CD95L system. Our data suggest an alternative explanation for observed cognitive deficits beyond an autoimmune explanation.

\section{Materials and Methods}

\section{Animals}

$\mathrm{BI} / 6-\mathrm{lpr}$ and $\mathrm{BI} / 6-$ gld mice were kindly provided by J Tschopp. C57BI/6 $(\mathrm{B} / / 6), \mathrm{CBA} / \mathrm{J}$ and $\mathrm{CBA} / \mathrm{J}-/ p r-c g$ mice were purchased from Jackson Laboratories (Bar Harbor, Maine, Germany).

\section{Cell and explant cultures}

Primary hippocampal neurons were cultured as previously reported. ${ }^{39}$ In brief, the hippocampi of E15 or P1 mice were dissected, trypsinized and physically dissociated. Cells were washed in HBSS and 150000 cells were plated onto poly-L-lysine (PLL)-treated glass coverslips in $6 \mathrm{~cm}$ Petri dishes containing Minimal essential medium (MEM) and 10\% heatinactivated horse serum. The cells were kept in $5 \% \mathrm{CO}_{2}$ at $36.5^{\circ} \mathrm{C}$. After $4 \mathrm{~h}$, the coverslips were transferred to a $6 \mathrm{~cm}$ dish containing MEM-N2 with B27 supplement (Gibco). For biochemical analysis 450000 cells were plated onto $3 \mathrm{~cm}$ tissue culture dishes containing MEM-N2 medium and B27 supplement and incubated in the same way as before. Hippocampal explants were plated on PLL-laminin-coated glass coverslips in 12-well plates containing MEM-N2 medium and B27 supplement. Cells were treated with the following reagents: anti-mouse Fas ligand (MFL3), antimouse Fas (Jo2) and hamster lgG (10 $\mu \mathrm{g} / \mathrm{ml})$, anti-human CD95L (NOK-1; $1 \mu \mathrm{g} / \mathrm{ml})$ and mouse $\mathrm{lgG}-1(1 \mu \mathrm{g} / \mathrm{ml})$, all purchased from Becton and Dickinson. LZ-CD95L was used at a concentration of $100 \mathrm{ng} / \mathrm{ml}{ }^{40}$ The pan-caspase inhibitor zVAD.fmk was used at $25 \mu \mathrm{M}$ and the MEK1 inhibitor (PD98059) was used at $200 \mathrm{nM}$. Neurons were incubated with the inhibitor 20 min (ZVAD.fmk) or $1 \mathrm{~h}$ (PD98059) prior to treatment with LZCD95L. Treatment with LZ-CD95L was performed at $4 \mathrm{~h}$ and 2 days after plating. For biochemical studies, cells were treated at $4 \mathrm{~h}$ after plating and 10 min prior to protein extraction. All cell lines were kept in $5 \% \mathrm{CO}_{2}$ at $36.5^{\circ} \mathrm{C}$.

\section{Cell analysis by flow cytometry}

To detect CD95 and CD95L expression on the cell surface of single cell suspensions from embryonic and postnatal brain $\left(5 \times 10^{5} \mathrm{cells} / \mathrm{ml}\right.$ in PBS), cells were fixed with $2 \%$ paraformaldehyde at $37^{\circ} \mathrm{C}$ for $15 \mathrm{~min}$. After blocking, cells were incubated with the primary antibodies (M20 or N20; Santa Cruz Biotechnology) $30 \mathrm{~min}$ on ice followed by the secondary antibody (30 min). Cytofluorometric analysis was performed on a FACScan (Becton Dickinson, Germany) using Cell Quest Software. A minimum of 10000 cells per sample was analyzed. For validation of the M20, N20 antibodies human melanoma tumors transfected with murine CD95L or empty vector and a thymoma cell line expressing CD95 were used.

\section{Histology}

Embryos (E16 and E18) and postnatal (P0, P5, P15 and P21) wt mice were transcardially perfused with $4 \%$ paraformaldehyde. Dissected brains were postfixed in the same fixative, and paraffin embedded. Sections of $5 \mu \mathrm{m}$ thickness were immunostained with rabbit antibodies against CD95 and CD95L (M20 and N20, respectively; Santa Cruz Biotechnology) and monoclonal antibody to calbindin (Sigma). For validation of the N20 antibody, human melanoma tumors transfected with murine CD95L or empty vector were used. Immunoreactive cells were only found in the CD95L-transfected and not in the vector-transfected tumors. After incubation with biotin-coupled secondary antibodies, sections were developed with $3^{\prime}, 3^{\prime}$-diaminobenzidine tetrahydrochloride (DAB; Sigma).

\section{Immunofluorescence}

Hippocampal cells or explants were fixed with $4 \%$ paraformaldehyde $37^{\circ} \mathrm{C}$ for $15 \mathrm{~min}$, quenched in $50 \mathrm{mM}$ ammonium chloride and extracted in $0.1 \%$ Triton X-100 for $5 \mathrm{~min}$. After blocking with blocking solution, cells were incubated with the following antibodies mouse anti-tubulin III $\beta$, rabbit antiCD95 (M20), rabbit CD95L (N20), mouse anti-NeuN, mouse anti-GFAP (Chemicon) or mouse anti-CNPase (Sigma). Immunoreactivities were visualized with a monoclonal or polyclonal antibody coupled to rhodamine or fluorescein isothiocyanate.

\section{Morphometric analyses of dendritic trees}

wt $(n=5), \operatorname{Ipr}(n=4)$ and gld $(n=6)$ postnatal mice (P5) were perfused with $4 \%$ paraformaldehyde in $0.1 \mathrm{M}$ phosphate buffer. The brains were dissected and postfixed in the same fixative. Thick hippocampal slices were obtained and small crystals of Dil were placed in the fimbria under the control of a dissection microscope. After 2 weeks, the slices were resectioned $(100 \mu \mathrm{m})$ with a vibratome. Sections were mounted with an antifading mounting medium, and Dil retrogradely labeled pyramidal neurons in the CA1 hippocampal region were selected for analysis. Confocal images were collected through the entire Z-axis using a Leica TCS 4D confocal scanning laser microscope. 3-D reconstructions of dendritic trees were obtained using the ImageJ program. The number of branching points, length of dendritic segments and length of dendritic arbors were measured using the ImageJ program (18-22 neurons per group). Statistical analyses were performed using the Student's $t$-test.

\section{Lentivirus infection}

Neurons were infected with the lentiviral vector $\mathrm{PEIGW}$ at an $\mathrm{MOI}$ of 5 . The plasmid pEIGW was constructed by replacing the eGFP sequence between the EF1 $\alpha$ promotor and the WPRE element in pWPTS-eGFP (kindly provided by D Trono, Geneva) with the IRES-eGFP cassette from pIRES2-eGFP (Clontech, Germany). All lentiviruses were propagated using previously described methods. ${ }^{41,42}$ Expression of all transgenes was confirmed in infected cells by flow cytometric analysis of GFP expression. The percentage of infected cells was $50-70 \%$. For the lentiviral gene delivery in vivo, pEIGW carrying human CD95L-GFP or GFP alone were injected into the $\mathrm{Cx}$. After 5 days, animals were perfused transcardially with $0.9 \% \mathrm{NaCl}$ solution followed by ice-cold $4 \%$ paraformaldehyde in PB. Brains were dissected and post-fixed overnight in the same solution and then embedded in paraffin.

\section{Detection of apoptosis}

To quantify DNA fragmentation, cells detached with trypsin were centrifuged at $200 \times g$ and fixed with $70 \%$ ethanol at $-20^{\circ} \mathrm{C}$ for $1 \mathrm{~h}$. Fixed cells were lyzed in a hypotonic lysis buffer $(0.1 \%$ sodium citrate and $0.1 \%$ Triton X-100) containing $50 \mu \mathrm{g} / \mathrm{ml}$ of propidium iodide, incubated at $4{ }^{\circ} \mathrm{C}$ overnight and analyzed by flow cytometry. ${ }^{43}$ Apoptosis in brain slices was detected by TUNEL as previously described. ${ }^{2}$ TUNEL-positive cells 
are expressed as relative to the total amount of cells, as detected by Hoechst staining.

\section{Protein extraction and immunoblotting}

Cells were harvested, washed in chilled phosphate-buffered saline (PBS), resuspended and homogenized in a buffer consisting of $0.2 \%$ SDS, phosphatase inhibitors ( $\mathrm{NaF}, \mathrm{NaN}_{3}$, pNPP NaPPi, $\beta$-glycerophosphate, $10 \mathrm{mM}$ each) and protease inhibitor (Complete, Roche). Lysates were centrifuged at $10000 \times g$ for $10 \mathrm{~min}$ at $4^{\circ} \mathrm{C}$ and boiled for $5 \mathrm{~min}$ in Laemmli's buffer. Proteins were analyzed by SDS-PAGE on 10 and $15 \%$ polyacrylamide gels and subsequently transferred to nitrocellulose. Nitrocellulose membranes were blotted with the following antibodies: anticleaved caspase-3 (Cell Signaling Technologies), anti-Tau-5 (Neo Markers) and anti-Tau-1 (Chemicon), anti phospho-ERK (Santa Cruz Biotechnology), anti ERK (Transduction Laboratories) and anti-actin (Sigma Aldrich). Immunoreactive proteins were visualized with peroxidase-conjugated anti-rabbit or anti-mouse antibodies (Santa Cruz Biotechnology) and enhanced chemiluminescence detection kit (Pierce Biotechnology) by exposure to Kodak X-Omat films.

\section{RNA isolation and affymetrix oligonucleotide array hybridization}

Total neuronal RNA was extracted using the RNeasy Mini Kit (Qiagen) and RNA quality was monitored by the Bioanalyzer 2100 RNA 6000 Nano assay (Agilent). In all, $1 \mu \mathrm{g}$ of total RNA was reverse transcribed essentially as described. ${ }^{44,45}$ Biotin-labeling during in vitro transcription from the T7 promoter using the ENZO RNA Transcript labeling kit was performed according to the Affymetrix protocol. cRNA samples were hybridized on Mouse Genome U74Av2 chips (MG-U74Av2), and data analyzed using the Affymetrix MAS 5.0 software package.

\section{Image and statistical analysis}

Neurons from in vitro cultures were photographed and analyzed using the $\mathrm{NIH} 1.62$ Image program. For the quantification of axon length, an individual axon was measured from the axon hillock to the most distal growth cone along a given route. The longest process of the neuron was considered the axon, as previously confirmed by Tau- 1 staining. This assumption was confirmed in previous experiments. For morphological measurements, at least 20 individual neurons per group were analyzed. The number of branches was quantified by defining the first point of divergence from the main neurites as secondary branches. The route of the axon was then followed along one of the secondary branches to the next point of divergence and similarly the number of these tertiary branches was counted. Quaternary and higher order branches were also identified and counted along the entire length of the axon. For the timelapse experiments, neurons treated with LZ-CD95L or control supernatant were recorded over $4 \mathrm{~h}$ with $4 \mathrm{~min}$ intervals. Gain of neurites in the neuron recorded after $4 \mathrm{~h}$ was quantified as extension. Loss of neurites after $4 \mathrm{~h}$ was counted as retraction. Neurites that changed their length were not included in the quantification. The axonal speed rate was quantified by subtracting the initial length to the final length of each particular axon in pixels per second. Axons were identified by their morphology.

Statistical analysis was performed using the Mann-Whitney test. All analyses were performed with the program package ADAM of the Biostatistics Unit of the German Cancer Research Center. Data from at least three independent experiments were used for statistical analysis.

\section{Acknowledgements}

We thank Inna Lavrik and Dagmar Riess for LZ-CD95L preparation, and Alexandra Beisel and Claudia Schmidt for technical assistance. We thank Jörg Tschopp for providing us with the Ipr and gld mutant mice. This work was supported by the Christopher Reeve Paralysis Foundation (Grant KAC2-0101-2), an SFB 405 Grant (DFG, Germany), and a SAF2001-3290 Grant (Spanish Ministry of Science and Technology).

\section{References}

1. Demjen D, Klussmann S, Kleber S, Zuliani C, Stieltjes B, Metzger C, Hirt UA, Walczak H, Falk W, Essig M, Edler L, Krammer PH and Martin-Villalba A (2004) Neutralization of CD95 ligand promotes regeneration and functional recovery after spinal cord injury. Nat. Med. 10: 389-395

2. Martin-Villalba A, Herr I, Jeremias I, Hahne M, Brandt R, Vogel J, Schenkel J, Herdegen T and Debatin KM (1999) CD95 ligand (Fas-L/APO-1L) and tumor necrosis factor-related apoptosis- inducing ligand mediate ischemia-induced apoptosis in neurons. J. Neurosci. 19: 3809-3817

3. Martin-Villalba A, Hahne M, Kleber S, Vogel J, Falk W, Schenkel J and Krammer PH (2001) Therapeutic neutralization of CD95L and TNF attentuates brain damage in stroke. Cell Death Differ. 8: 679-686

4. Kischkel FC, Hellbardt S, Behrmann I, Germer M, Pawlita M, Krammer PH and Peter ME (1995) Cytotoxicity-dependent APO-1 (Fas/CD95)-associated proteins form a death-inducing signaling complex (DISC) with the receptor. EMBO J. 14: 5579-5588

5. Medema JP, Scaffidi C, Kischkel FC, Shevchenko A, Mann M, Krammer PH and Peter ME (1997) FLICE is activated by association with the CD95 deathinducing signaling complex (DISC). EMBO J. 16: 2794-2804

6. Boldin MP, Varfolomeev EE, Pancer Z, Mett IL, Camonis JH and Wallach D (1995) A novel protein that interacts with the death domain of Fas/APO1 contains a sequence motif related to the death domain. J. Biol. Chem. 270: 7795-7798

7. Scaffidi C, Fulda S, Srinivasan A, Friesen C, Li F, Tomaselli KJ, Debatin KM, Krammer PH and Peter ME (1998) Two CD95 (APO-1/Fas) signaling pathways. EMBO J. 17: 1675-1687

8. Desbarats J, Birge RB, Mimouni-Rongy M, Weinstein DE, Palerme JS and Newell MK (2003) Fas engagement induces neurite growth through ERK activation and p35 upregulation. Nat. Cell Biol. 5: 118-125

9. Park C, Sakamaki K, Tachibana O, Yamashima T, Yamashita J and Yonehara $S$ (1998) Expression of fas antigen in the normal mouse brain. Biochem. Biophys. Res. Commun. 252: 623-628

10. Shin DH, Lee E, Kim HJ, Kim S, Cho SS, Chang KY and Lee WJ (2002) Fas ligand mRNA expression in the mouse central nervous system. J. Neuroimmunol. 123: 50-57

11. Cheema ZF, Wade SB, Sata M, Walsh K, Sohrabji F and Miranda RC (1999) Fas/Apo [apoptosis]-1 and associated proteins in the differentiating cerebral cortex: induction of caspase-dependent cell death and activation of NFkappaB. J. Neurosci. 19: 1754-1770

12. Cheema ZF, Santillano DR, Wade SB, Newman JM and Miranda RC (2004) The extracellular matrix, p53 and estrogen compete to regulate cell-surface Fas/Apo-1 suicide receptor expression in proliferating embryonic cerebral cortical precursors, and reciprocally, Fas-ligand modifies estrogen control of cell-cycle proteins. BMC Neurosci. 5: 11

13. Ceccatelli S, Tamm C, Sleeper E and Orrenius S (2004) Neural stem cells and cell death. Toxicol. Lett. 149: 59-66

14. Ricci-Vitiani L, Pedini F, Mollinari C, Condorelli G, Bonci D, Bez A, Colombo A, Parati E, Peschle C and De Maria R (2004) Absence of caspase 8 and high expression of PED protect primitive neural cells from cell death. J. Exp. Med. 200: $1257-1266$

15. Oppenheim RW, Flavell RA, Vinsant S, Prevette D, Kuan CY and Rakic P (2001) Programmed cell death of developing mammalian neurons after genetic deletion of caspases. J. Neurosci. 21: 4752-4760

16. Cohen PL and Eisenberg RA (1992) The Ipr and gld genes in systemic autoimmunity: life and death in the Fas lane. Immunol Today 13: 427-428 
17. Kovac AD, Grammig J, Mahlo J, Steiner B, Roth K, Nitsch R and Bechmann I (2002) Comparison of neuronal density and subfield sizes in the hippocampus of CD95L-deficient (gld), CD95-deficient (lpr) and nondeficient mice. Eur. J. Neurosci. 16: 159-163

18. Adachi M, Watanabe-Fukunaga $R$ and Nagata $S$ (1993) Aberrant transcription caused by the insertion of an early transposable element in an intron of the Fas antigen gene of Ipr mice. Proc. Natl. Acad. Sci. USA 90: 1756-1760

19. Watanabe-Fukunaga R, Brannan $\mathrm{Cl}$, Copeland NG, Jenkins NA and Nagata S (1992) Lymphoproliferation disorder in mice explained by defects in Fas antigen that mediates apoptosis. Nature 356: 314-317

20. Cleveland DW, Hwo SY and Kirschner MW (1977) Purification of tau, a microtubule-associated protein that induces assembly of microtubules from purified tubulin. J. Mol. Biol. 116: 207-225

21. Drubin DG and Kirschner MW (1986) Tau protein function in living cells. J. Cell Biol. 103: 2739-2746

22. Schneider A, Biernat J, von Bergen M, Mandelkow E and Mandelkow EM (1999) Phosphorylation that detaches tau protein from microtubules (Ser262, Ser214) also protects it against aggregation into Alzheimer paired helical filaments. Biochemistry 38: 3549-3558

23. Schwab C, DeMaggio AJ, Ghoshal N, Binder LI, Kuret J and McGeer PL (2000) Casein kinase 1 delta is associated with pathological accumulation of tau in several neurodegenerative diseases. Neurobiol. Aging 21: 503-510

24. Smith HR and Steinberg AD (1983) Autoimmunity - a perspective. Ann. Rev. Immunol. 1: 175-210

25. Sakic B, Szechtman H, Denburg JA, Gorny G, Kolb B and Whishaw IQ (1998) Progressive atrophy of pyramidal neuron dendrites in autoimmune MRL-Ipr mice. J. Neuroimmunol. 87: 162-170

26. Raoul C, Henderson CE and Pettmann B (1999) Programmed cell death of embryonic motoneurons triggered through the Fas death receptor. J. Cell Biol. 147: 1049-1062

27. Holler N, Zaru R, Micheau O, Thome M, Attinger A, Valitutti S, Bodmer JL, Schneider P, Seed B and Tschopp J (2000) Fas triggers an alternative, caspase-8-independent cell death pathway using the kinase RIP as effector molecule. Nat. Immunol. 1: 489-495

28. Wagner U, Utton M, Gallo JM and Miller CC (1996) Cellular phosphorylation of tau by GSK-3 beta influences tau binding to microtubules and microtubule organisation. J. Cell Sci. 109: 1537-1543

29. Richter JD (2001) Think globally, translate locally: what mitotic spindles and neuronal synapses have in common. Proc. Natl. Acad. Sci. USA 98: 7069-7071

30. Takahashi T, Tanaka M, Brannan Cl, Jenkins NA, Copeland NG, Suda T and Nagata S (1994) Generalized lymphoproliferative disease in mice, caused by a point mutation in the Fas ligand. Cell 76: 969-976

31. Sneller MC, Straus SE, Jaffe ES, Jaffe JS, Fleisher TA, Stetler-Stevenson M and Strober W (1992) A novel lymphoproliferative/autoimmune syndrome resembling murine Ipr/gld disease. J. Clin. Invest. 90: 334-341
32. Hess DC, Taormina M, Thompson J, Sethi KD, Diamond B, Rao R Chamberlain CR and Feldman DS (1993) Cognitive and neurologic deficits in the MRL/pr mouse: a clinicopathologic study. J. Rheumatol. 20: 610-617

33. Feinglass EJ, Arnett FC, Dorsch CA, Zizic TM and Stevens MB (1976) Neuropsychiatric manifestations of systemic lupus erythematosus: diagnosis, clinical spectrum, and relationship to other features of the disease. Medicine (Baltimore) 55: 323-339

34. Johnson RT and Richardson EP (1968) The neurological manifestations of systemic lupus erythematosus. Medicine (Baltimore) 47: 337-369

35. Denburg SD and Denburg JA (2003) Cognitive dysfunction and antiphospholipid antibodies in systemic lupus erythematosus. Lupus 12: 883-890

36. Denburg JA, Carbotte RM and Denburg SD (1987) Neuronal antibodies and cognitive function in systemic lupus erythematosus. Neurology 37: 464-467

37. Denburg SD, Carbotte RM, Ginsberg JS and Denburg JA (1997) The relationship of antiphospholipid antibodies to cognitive function in patien1ts with systemic lupus erythematosus. J. Int. Neuropsychol. Soc. 3: 377-386

38. Denburg JA, Carbotte RM and Denburg SD (1987) Neuronal antibodies and cognitive function in systemic lupus erythematosus. Neurology 37: 464-467

39. Banker G and Goslin K (1988) Developments in neuronal cell culture. Nature 336: 185-186

40. Walczak H, Miller RE, Ariail K, Gliniak B, Griffith TS, Kubin M, Chin W, Jones J, Woodward A, Le T, Smith C, Smolak P, Goodwin RG, Rauch CT, Schuh JC and Lynch DH (1999) Tumoricidal activity of tumor necrosis factor-related apoptosis- inducing ligand in vivo [see comments]. Nat. Med. 5: 157-163

41. Naldini L, Blomer U, Gage FH, Trono D and Verma IM (1996) Efficient transfer, integration, and sustained long-term expression of the transgene in adult rat brains injected with a lentiviral vector. Proc. Natl. Acad. Sci. USA 93: 11382-11388

42. Zufferey R, Dull T, Mandel RJ, Bukovsky A, Quiroz D, Naldini L and Trono D (1998) Self-inactivating lentivirus vector for safe and efficient in vivo gene delivery. J. Virol. 72: 9873-9880

43. Nicoletti I, Migliorati G, Pagliacci MC, Grignani F and Riccardi C (1991) A rapid and simple method for measuring thymocyte apoptosis by propidium iodide staining and flow cytometry. J. Immunol. Methods 139: 271-279

44. Ernst T, Hergenhahn M, Kenzelmann M, Cohen CD, Bonrouhi M, Weninger A, Klaren R, Grone EF, Wiesel M, Gudemann C, Kuster J, Schott W, Staehler G, Kretzler M, Hollstein M and Grone HJ (2002) Decrease and gain of gene expression are equally discriminatory markers for prostate carcinoma: a gene expression analysis on total and microdissected prostate tissue. Am. J. Pathol. 160: 2169-2180

45. Kenzelmann M, Klaren R, Hergenhahn M, Bonrouhi M, Grone HJ, Schmid W and Schutz G (2004) High-accuracy amplification of nanogram total RNA amounts for gene profiling. Genomics 83: 550-558

\section{Supplementary Information accompanies the paper on Cell Death and Differentiation website (http://www.nature.com/cdd)}

